\title{
Multi-objective Transportation Problem with Cost Reliability Under Uncertain Environment
}

\author{
Gurupada Maity ${ }^{1}$, Sankar Kumar Roy ${ }^{2 *}$, and José Luis Verdegay ${ }^{3}$ \\ ${ }^{1,2}$ Department of Applied Mathematics with Oceanology and Computer Programming \\ Vidyasagar University, Midnapore-721102, West Bengal, India \\ E-mail:maity.g0795@gmail.com,sankroy2006@gmail.com \\ ${ }^{3}$ Department of Computer Science and Artificial Intelligence \\ University of Granada, Granada, Spain \\ Email:verdegay@decsai.ugr.es
}

Received 28 January 2016

Accepted 3 May 2016

\begin{abstract}
This paper analyzes the study of a Multi-Objective Transportation Problem (MOTP) under uncertain environment. Assuming the uncertainty in real-life decision making problems, the concept of reliability is incorporated in the transportation cost and the effectiveness is justified through the proposed MOTP. Again, considering the real phenomenon in the MOTP, we consider the transportation parameters, like as supply and demand as uncertain variables. Also, we consider the fuzzy multi-choice goals to the objective functions of the MOTP; and Fuzzy Multi-Choice Goal Programming (FMCGP) is used to select the proper goals to the objective functions of the proposed MOTP. Here, the proposed study is not only confined to obtain the compromise solution but also to fix up the proper goals to the objective functions of the MOTP. A numerical example is presented to illustrate and justify the proposed study. Finally, the paper ends with the conclusion and future study.
\end{abstract}

Keywords: Transportation problem, Multi-objective decision making, Reliability, Uncertain programming, Fuzzy multi-choice goal programming.

\section{Introduction}

The Transportation Problem (TP) is a special type of decision making problem which may be considered as the central nerve system to keep the balance in economical world from ancient day to today. The TP can be treated as a special case of linear programming problem and its model is used to determine how many units of items to be shipped from each origin to various destinations, satisfying source availabilities and destination demands, while minimizing the total cost of transportation along with cutting down the costs per unit items for the purchasers.
The basic TP was originally developed by Hitchcock [1] and later independently developed by Koopmans [2]. The present competitive market scenario suggests that the single objective TP is not sufficient to handle the real-life decision making problem. To cover the real-life situations on TP, we introduce here the multiobjective concept on TP in which the objective functions are conflicting to each other. Charnes and Cooper [3] first discussed various approaches on the solution of managerial level problems involving multiple conflicting objective functions. Garfinkel and Rao [4] worked out two objective functions by giving high and low priorities to the objective functions.

* Corresponding author. 
Cadenas and Verdegay [5] solved multi-objective linear programming problem by fuzzy ranking function. Waiel [6] developed the MOTP under fuzziness to obtain the compromise solution. Mahapatra et al. ([7], [8]) studied on multi-objective TP under different stochastic environments. Gupta and Kumar [9], Ebrahimnejad [10] worked on uncertainty under fuzzy environments. Jiménez and Verdegay [11] used the fuzzy uncertainty in solving solid transportation problem. A number of studies has been done by several researchers such as Delgado et al. [12], Mahapatra et al. [13], Maity and Roy [14], Midya and Roy [15], Roy et al. [16], and many others for solving MOTP problems under different uncertain environments.

The parameters of the TP such as the supply, the demand and the cost parameters are not precise due to the complex real world problems. To analyze the TP in the complex ground, here we treat the supply and the demand parameters as uncertain variables, and the transportation cost is analyzed through reliability. Due to damage of transporting goods for delay of transportation within the schedule time or loss of wealth due to transportation before schedule time, as a result customers are affected. To remove this factor, we introduce the reliability in the cost parameters of the TP. Again, the study of MOTP in which the objective functions are conflicting type, the reliability in cost penalty may cause to increase the cost of transportation or increase the values of objective functions which are minimization type and to decrease the profit of DM or decrease the value of objective functions which are maximization type. When the cost reliability factor is introduced as the rate of damage property which creates a diverse relation for both type of objective functions, but how much amount of loss caused by the factor is totally independent to the objective functions. So, it is clear that the reliability in cost parameter preserves the conflicting nature to the objective functions of the MOTP. Major studies in the ground of the MOTP, where the uncertainties are taken into consideration through fuzzy, stochastic, interval etc. for the transportation cost, the demand, and the supply parameters in the TP but here for the first time in the MOTP, we analyze the uncertainty into the cost parameter of MOTP using reliability.

Many researchers have shown great interest on solving real life uncertain mathematical programming problems. A few references are presented with their works. In order to deal with human uncertainty, Liu [17] presented a study on uncertainty theory and later, it was defined by Liu ([18], [19]) based on normality, duality, sub-additivity and product axioms. Gao [20] introduced some properties on continuous uncertain measure in his paper. In practical aspect, Liu [21] proposed an uncertain mathematical programming involving uncertain variables.

Considering the situations of real-life decision making problems, we design this paper on the TP in multiobjective ground where the objective functions are conflicting. Many situations occurred where the solution of a MOTP is found as compromise solution, but the solution often depends on the weights of objective functions proposed by the DM. Then in the MOTP the compromise solution satisfying the goals of the objective functions which plays an effective role for solving it. In this case, we propose goals to each objective functions of the MOTP. It is not always true that the solution of the MOTP will be specified as single choice of goals of the DM, because in case, it works in favour of DM but it is not so favoured for customers. So, the DM needs to consider a multiple choice of goals corresponding to the objective functions and to select the best goals through the solution. Again, we consider the goals to the objective functions as multi-choice fuzzy numbers to accommodate the real life decision making situations. Goal Programming (GP) is one of the most useful and well known decision making technique which was introduced by Charnes et al. [22]. The interesting philosophy and high applicability of GP in handling real world decision making problems with multi-objective structures made it very useful and widespread. This leads to further development of GP for different decision making problems. A lot of works based on goal programming has been done by several researchers such as Chang [23], Liao [24], Lee [25], Maity and Roy [26], Narasimhan [27], Tamiz et al. [28] and many others. Study on multi-choice goals has been done by several researchers e.g., Chang [29], Tabrizi [30], Roy [31], Maity and Roy [32], Roy et al. [33] and many others.

The main aim of this paper is to study the MOTP in which the objective functions are conflicting and each of them has fuzzy multi-choice goals. Also, considering the present market scenario, we incorporate the concept of cost reliability into the transportation cost, and the demand and the supply parameters are treated as the uncertain variables. The methodologies are presented to tackle the situations and to predict the optimal goals of the objective functions as well as the optimal solution of the MOTP.

The rest of the paper is organized as follows: In Sections 2 and 3 , the problem background and mathematical models are described for the MOTP. Section 4 contains the solution procedure including the discussion on the MCGP and the FMCGP and reduces 
the uncertainty in the MOTP. We demonstrate the usefulness of the proposed model with a realistic example in the MOTP in Section 5. In Section 6, we present the sensitivity analysis regarding the numerical example. Finally, in Section 7, conclusion with future study and limitations are presented related to our consideration.

\section{Problem Background}

In the TP, the completion time of transportation of amount of goods should be finished within the specified time, otherwise there may be created a damage of the items or storing problem and/or the customer may reject the ordered item. In that situation, the transportation cost or the profit may not be considered as crisp value. Then the selection of goals for the objective functions or the solution of the MOTP cannot be made in usual way. To overcome this difficulty by selecting the proper goals to the objective functions, here, we incorporate the concept of reliability for the cost parameters in the TP. In that situation, we introduce a new term "cost reliability" for the transportation cost in the proposed study.

Generally, Reliability (Richard et al. [34]) refers the probability of a machine operating its intended purpose adequately for the period of time desired under the operating conditions encountered. More precisely, reliability is the probability with which the devices will not fail to perform a required operation for a certain period of time.

Definition 2.1(Cost Reliability): Cost reliability is the probability that the transportation of goods will not fail to complete the transporting of goods in the schedule time, which creates a probabilistic cost in the transportation problem. The probabilistic cost in the transportation problem increases the original value of transportation of goods and simultaneously makes a difference in profit margin.

We assume the failure rate, $\lambda$ by the ratio of due time or over time, $\delta \tau$ to complete the transportation of goods; and the total estimated time $\mathrm{T}$ of transportation i.e., $\lambda=\frac{\delta \tau}{\tau}$, where $\boldsymbol{\tau}$ represents the amount of late or early transportation time corresponding to the schedule time of transportation.

Here, we define the cost reliability $\mathrm{R}(\boldsymbol{\tau})$, which is a function of time $\boldsymbol{\tau}$ as follows:

$\mathrm{R}(\tau)=$

Amount of goods remains in good condition due to loss of tims Total amount of goods
The probability of failure, $\mathrm{Q}(\tau)$ can be expressed as follows:

$\mathrm{Q}(\tau)=\frac{\text { Amount of goods damaged due or over time }}{\text { Total amount of goods }}$
Clearly, $\quad \mathrm{R}(\tau)+\mathrm{Q}(\tau)=1$, so, $\mathrm{Q}(\tau)=1-\mathrm{R}(\tau)$.

Therefore, $R(\tau)=e^{-\int_{0}^{\tau} \lambda d \tau}$. Assuming that the failure rate, $\lambda$ is a constant in respect to time $\tau$, then we have $R(\tau)=e^{-\lambda \tau}$. And finally, $\mathrm{Q}(\tau)$ can be found as $\mathrm{Q}(\tau)=1$ $R(\tau)=1-e^{-\lambda \tau}$.

To analyze the proposed study with a better impact in reality, we consider the parameter, $\lambda$ is the ratio of a function of decision variable, $x_{i j}$ and $a_{i}$, is the constant supply of the $i^{\text {th }}$ source, i.e., $\lambda=\frac{a_{i j} j}{a_{i}}$. When the value of $\lambda$ increases the value of reliability $R(\tau)$ decreases which means that if the amount of transported goods becomes larger, then the amount of items may be defective in bigger rate. Again the value of $R(\tau)=e^{-\lambda \tau}$ depends on the time $\tau$, then we would like to consider the time $\tau$ as the expected loss of time to complete the work. If the transportation made in time, then $\tau=0$, so reliability value is maximized, i.e., $R(\tau)=1$. Again it is true that in the TP, some variables may take value zero. It means no item is transported in that route. So the reliability is again equal to 1 for this path in the proposed model which does not create any complexity to take the decision for the DM.

Taking advantage of the reliability function in the reallife decision making problem, we formulate the MOTP where the objective functions are conflicting and connected with some multi-choice goals. The way of formulating the mathematical model of MOTP using cost reliability is included in detail in the next section.

\section{Mathematical Model}

In the traditional TP, a homogenous product is to be transported from several origins (or sources) to numerous destinations in such way that the total transportation cost is minimized. Suppose there are $m$ origins $(i=1,2, \ldots, m)$ and $n$ destinations $(j=1,2, \ldots, n)$. The sources may be production facilities, warehouses etc. and these are characterized by available supplies $a_{1}, a_{2}, \ldots, a_{m}$. The destinations may be warehouses and sales outlets etc., and these are also characterized by demand levels $b_{1}, b_{2}, \ldots, b_{n}$. The transportation cost $C_{i j}$ is associated with transporting a unit of product from the origin $i$ to the destination $j$. A variable $x_{i j}$ is used to represent the unknown quantity to be transported from the origin $\mathrm{O}_{\mathrm{i}}$ to the destination $\mathrm{D}_{\mathrm{j}}$. 
The mathematical model of a classical transportation problem is as follows:

\section{Model 1}

$$
\begin{array}{cl}
\text { minimize } & \mathrm{Z}=\sum_{\mathrm{i}=1}^{\mathrm{m}} \sum_{\mathrm{j}=1}^{\mathrm{n}} \mathrm{C}_{\mathrm{ij}} \mathrm{x}_{\mathrm{ij}}, \\
\text { subject to } & \sum_{\mathrm{j}=1}^{\mathrm{n}} \mathrm{x}_{\mathrm{ij}} \leq \mathrm{a}_{\mathrm{i}}(\mathrm{i}=1,2, \ldots, \mathrm{m}), \\
& \sum_{\mathrm{i}=1}^{\mathrm{m}} \mathrm{x}_{\mathrm{ij}} \geq \mathrm{b}_{\mathrm{j}}(\mathrm{j}=1,2, \ldots, \mathrm{n}), \\
& \mathrm{x}_{\mathrm{ij}} \geq 0 \forall i, j .
\end{array}
$$

The feasibility condition is considered as: $\sum_{\mathrm{i}=1}^{\mathrm{m}} \mathrm{a}_{\mathrm{i}} \geq \sum_{\mathrm{j}=1}^{\mathrm{n}} \mathrm{b}_{\mathrm{j}}$.

According to the challenging competitive market scenario, several objective functions related to a transportation problem like minimizing the transportation cost, maximizing the transportation profit, minimizing the toll tax etc. are required. Again, there is no connection between the cost parameters in the different objective functions of the TP, so, they are considered as conflicting and commensurable to each other. In multi-objective environment of the TP, i.e., the MOTP can be defined as follows:

\section{Model 2}

minimize/ maximize

$$
\mathrm{Z}^{\mathrm{t}}=\sum_{\mathrm{i}=1}^{\mathrm{m}} \sum_{\mathrm{j}=1}^{\mathrm{n}} \mathrm{C}_{\mathrm{ij}}^{\mathrm{t}} \mathrm{x}_{\mathrm{ij}}(\mathrm{t}=1,2, \ldots, \mathrm{r})
$$

subject to

$$
\sum_{\mathrm{j}=1}^{\mathrm{n}} \mathrm{x}_{\mathrm{ij}} \leq \mathrm{a}_{\mathrm{i}}(\mathrm{i}=1,2, \ldots, \mathrm{m})
$$$$
\sum_{i=1}^{m} x_{i j} \geq b_{j}(j=1,2, \ldots, n) \text {, }
$$$$
x_{i j} \geq 0 \forall i, j \text {. }
$$

Here, $C_{i j}^{t}, a_{i}$ and $b_{j}$ are the transportation cost, the supply and the demand parameters respectively for the $t^{\text {th }}$ objective function in the MOTP.

In Model 2, the quantities $\mathrm{C}_{\mathrm{ij}}^{\mathrm{t}}, \mathrm{a}_{\mathrm{i}}$ and $\mathrm{b}_{\mathrm{j}}$ are all assumed to be crisp numbers. However, if sometimes, the transportation plan is made in advance, so the quantities are not generally fixed but approximate amounts of these are obtained from practical experience or expert knowledge. In this case, we may assume the quantities are uncertain variables. Then the transportation Model 2 is only a conceptual model rather than a mathematical model because there does not exist a natural order-ship in the complex world. A large number of decision making problems has been solved by several researchers in which uncertain situation is directly introduced in the parameters of the MOTP, but here, we introduce the uncertainty through reliability by considering due or early transportation time of delivering the goods. Due to the late or early reach of the transporting goods, the customer or the store keeper fails to manage it. So, the DM should consider to his mind the matter and as a whole the optimum value of the objective functions are affected. Considering this situation, we introduce time in the cost parameter which reduces the cost parameter of the MOTP to the cost parameter with reliability. When the cost reliability is considered for all the objective functions then time is taken as independent to each other and the conflicting nature of the objective functions preserved in the MOTP. Again, the supply and demand quantities are not crisp due to weather condition, seasonal effect, market situation etc. So, the uncertain measure (here denoted as $\mathcal{M}$ ) from an uncertainty distribution for supply and demand constraints are considered. Then Model 2 reduces to the following mathematical model (Model 3) as follows:

\section{Model 3}

minimize/maximize

$$
\mathrm{Z}^{\mathrm{t}}=\sum_{\mathrm{i}=1}^{\mathrm{m}} \sum_{\mathrm{j}=1}^{\mathrm{n}} \mathrm{R}\left(\mathrm{C}_{\mathrm{ij}}^{\mathrm{t}}\right) \mathrm{x}_{\mathrm{ij}} \quad(\mathrm{t}=1,2, \ldots, \mathrm{r}),
$$

subject to

$$
\begin{aligned}
& M\left(\sum_{j=1}^{n} x_{i j} \leq a_{i}\right) \geq \gamma_{i} \quad(i=1,2, \ldots, m), \\
& M C\left(\sum_{i=1}^{m} x_{i j} \geq b_{j}\right) \geq \delta_{j} \quad(j=1,2, \ldots, n), \\
& x_{i j} \geq 0 \forall i, j .
\end{aligned}
$$

It is assumed that $a_{i}>0, b_{j}>0$ and the specified stochastic levels or predetermined confidence levels are defined as $0<\gamma_{i}<1, \forall i$, and $0<\delta_{j}<1, \forall j$. In Model 3, $\mathrm{R}\left(\mathrm{C}_{\mathrm{ij}}^{\mathrm{t}}\right)$ is considered as cost parameter under reliability. The delay of supply of items causes to damage the items, in this case the value of profit function (maximization type) decreases and the penalty cost due to loss of time is considered when the objective function is of minimization type (like transportation cost) which increases the value of the respective objective function. Then $\mathrm{R}\left(\mathrm{C}_{\mathrm{ij}}^{\mathrm{t}}\right)$ takes the following form of cost parameter as:

$$
R\left(C_{i j}^{t}\right)=C_{i j}^{t}+C_{i j}^{t}\left(1-R_{i j}^{t}\right) \text {, }
$$

for the objective function is of minimization type and $R\left(C_{i j}^{t}\right)=C_{i j}^{t}-C_{i j}^{t}\left(1-R_{i j}^{t}\right)$,

for the objective function is of maximization type.

Here $R_{i j}^{t}$ is the reliability function of the $t^{\text {th }}$ objective function for the $(i, j)$-th node which depends on fixed time $(\tau)$, decision variable $\left(\mathrm{x}_{\mathrm{ij}}\right)$ and demand $\left(\mathrm{a}_{\mathrm{i}}\right)$. For consistency of reliability in each node, the DM measures the time $(\tau)$ in a unit scale, otherwise there may occur large deviations in the cost values and produces an optimal solution, which is not significantly a good result. 
Many real-life MOTPs, the DM wishes to solve the objective functions by considering certain goals to the objective functions, but how the goals to be chosen which is a complex task to the DM. To overcome this difficulty, we consider the fuzzy multi-choice goals with the respective deviations corresponding to each objective function. In that situation, the DM wishes to fix the goals in such a way that the compromise solution becomes a better solution corresponding to the chosen goals. Let us consider the fuzzy multi-choice goals $\hat{g}_{\mathrm{t}}^{1}, \hat{g}_{\mathrm{t}}^{2}, \ldots, \hat{g}_{\mathrm{t}}^{p}(\mathrm{t}=1,2, \ldots, \mathrm{r})$ for the $t^{\text {th }}$ objective function. Then each of the objective functions in the mathematical Model 3, there are multi-choice fuzzy goals, so, we present the procedure to select the optimum goals and get the optimal solution in the next section as a solution procedure.

\section{Solution Procedure}

A brief introduction of Multi-Choice Goal Programming (MCGP) is presented in first subsection. Later, we present the reduction procedure of uncertainty in the MOTP. Finally, we present the solution procedure of the MOTP with fuzzy multi-choice goals.

\subsection{Multi-choice goal programming}

In goal programming literature, Chang [2] first proposed the MCGP approach which allows the DM to set MultiChoice Aspiration Levels (MCALs) for each goal (i.e., one goal is mapping with multiple aspiration levels). The general formulation of goal programming (GP) can be written as follows:

$$
\text { GP: } \begin{array}{ll}
\text { minimize } & \sum_{\mathrm{t}=1}^{\mathrm{r}} \mathrm{w}_{\mathrm{t}}\left|\mathrm{Z}^{\mathrm{t}}(\mathrm{x})-\mathrm{g}_{\mathrm{t}}\right| \\
\text { subject to } & \mathrm{x} \in F,
\end{array}
$$

where $\mathrm{F}$ is the feasible set and $\mathrm{w}_{\mathrm{i}}$ are the weights attached to the deviation of the achievement function $\mathrm{Z}^{\mathrm{t}}(\mathrm{x})$, which is the $\mathrm{t}^{\text {th }}$ objective function of the $\mathrm{t}^{\text {th }}$ goal and $\mathrm{g}_{\mathrm{t}}$ is the aspiration level of the $\mathrm{t}^{\text {th }}$ goal. $\left|Z^{t}(x)-g_{t}\right|$ represents the deviation of the $t^{\text {th }}$ goal. Later on, a modification on GP is provided and is denoted as weighted goal programming (WGP).

According to the real life decision making problem, the goals of the objective functions cannot be predicted as crisp values always, they may be considered as fuzzy goals. So the goals are fuzzy multi-choice goals corresponding to the objective functions. Under the environment of FMCGP the formulation of GP reduces to the following form:

\section{FMCGP:}

$$
\text { minimize } \quad \sum_{\mathrm{t}=1}^{\mathrm{r}} \mathrm{w}_{\mathrm{t}} \mid \mathrm{Z}^{\mathrm{t}}(\mathrm{x})-\hat{g}_{\mathrm{t}}^{1} \text { or } g_{\mathrm{t}}^{2} \text { or } \ldots . . \text { or } \hat{g}_{\mathrm{t}}^{p} \mid
$$$$
\text { subject to } \quad \mathrm{x} \in F \text {. }
$$

Here, the aspiration levels, $\hat{g}_{t}^{p} \forall p, t$ are assumed to be triangular fuzzy numbers with membership functions, $\mu_{t}^{p} \forall p, t$.

\subsection{Reduction of uncertainty in the MOTP}

Here, we introduce the concept of uncertainty distribution in order to describe the uncertain variable. Due to insufficient information of demand and supply in the MOTP, we incorporate the uncertainty in the constraints. However, in our proposed study, we consider the demands and the supply parameters as Normal distribution. There are several ways to tackle the uncertain constraints, here we use the uncertain measure proposed by Liu (2009, ch.7). Let us introduce some useful definitions; and theorem about on the uncertain variable.

According to our assumption that the supply and demand parameters in Model 3 are taken as uncertain measure (here denoted as $\boldsymbol{M}$ ) from an uncertainty distribution for supply and demand, so we define uncertain distribution function as follows:

Definition 4.2.2.: (Liu (2007, ch. 4)) Let $\xi$ be an uncertain variable. Then the uncertainty distribution denoted as $\Phi$ of $\xi$ is defined by $\Phi(\mathrm{x})=\mathcal{M}\{\xi \leq \mathrm{x}\}$ for any real number $\mathbf{x}$.

Without loss of generality, we may consider the Normal distribution for the supply and demand parameters in Model 3. A Normal uncertain distribution function and its inverse function is considered as follows:

Definition 4.2.3.: An uncertain variable $\xi$ is called Normal if it has a Normal uncertain distribution

$$
\Phi(\mathrm{x})=\left[1+\exp \left(\frac{\pi(\theta-x)}{\sqrt{3} \sigma}\right)\right]^{-1}
$$

for any real number $\mathrm{x}$, which is denoted by $N(e, \sigma)$ where $\operatorname{and} \sigma$ are real numbers with $\sigma>0$.

Definition 4.2.4.: (Liu (2007, ch. 4)) Let $\xi$ be an uncertain variable with regular uncertainty distribution $\Phi(\mathrm{x})$. Then the inverse function $\Phi^{-1}(\alpha)$ is called the inverse uncertainty distribution of $\xi$, for any real number $\alpha$.

As the supply and demand parameters are uncertain variable, so, uncertain measure is introduced in the supply and demand constraints of Model 3. These 
constraints are reduced into the equivalent crisp forms by using the following measure inversion theorem.

Theorem 4.2.1.: (Measure Inversion Theorem (Liu (2007, ch. 4)) Let $\xi$ be an uncertain variable corresponding to uncertain distribution $\Phi$. Then for any real number $\mathrm{x}$, we have,

$\Phi(\mathrm{x})=\mathcal{M}\{\xi \leq \mathrm{x}\}$ then $\mathcal{M}\{\xi \geq \mathrm{x}\}=1-\Phi(\mathrm{x})$.

The inverse uncertainty distribution of Normal uncertain variable $N(\Theta, \sigma)$ is defined as follows:

$\Phi^{-1}(\alpha)=\mathrm{e}+\frac{\sqrt{a} \sigma}{\pi} \ln \frac{\alpha}{1-\alpha}$,

where $\ln$ denotes natural logarithm.

The Model 3 is not a deterministic form due to presence of uncertain variable in the constraints. Assuming that $a_{i}(i=1,2, \ldots, m)$ and $b_{j}(j=1,2, \ldots, n)$ are independent uncertain variables with uncertainty distributions $\theta_{i}(\mathrm{i}=1,2, \ldots, \mathrm{m})$ and $\Psi_{j}(\mathrm{j}=1,2, \ldots, \mathrm{n})$ respectively. Then the measure inversion theorem is provided the following results:

$$
\begin{aligned}
& \mathcal{M}\left(\sum_{j=1}^{n} x_{i j} \leq a_{i}\right) \geq \gamma_{i} \quad \text { is } \quad \text { equivalent to } \\
& \sum_{j=1}^{n} x_{i j} \leq \theta_{i}^{-1}\left(1-\gamma_{i}\right), \\
& \text { and } \\
& \mathcal{M}\left(\sum_{i=1}^{m} x_{i j} \geq b_{j}\right) \geq \delta_{j} \text { is reduced to } \\
& \sum_{j=1}^{n} x_{i j} \geq \Psi_{j}^{-1} \delta_{j}, \text { for } i=1,2, \ldots, m ; j=1,2, \ldots, n .
\end{aligned}
$$

Using above results, Model 3 is equivalent to the following model:

\section{Model 4}

$$
\begin{aligned}
& \mathrm{Z}^{\mathrm{t}}=\sum_{\mathrm{i}=1}^{\mathrm{m}} \sum_{\mathrm{j}=1}^{\mathrm{n}}\left[\mathrm{C}_{\mathrm{ij}}^{\mathrm{t}} \pm \mathrm{C}_{\mathrm{ij}}^{\mathrm{t}}\left(1-\mathrm{R}_{\mathrm{ij}}^{\mathrm{t}}\right)\right] \mathrm{x}_{\mathrm{ij}}(\mathrm{t}=1,2, \ldots, \mathrm{r}), \\
& \text { subject to } \\
& \sum_{j=1}^{n} \mathrm{x}_{i j} \leq \theta_{\mathrm{i}}^{-1}\left(1-\gamma_{i}\right) \quad(i=1,2, \ldots, m), \\
& \sum_{\mathrm{i}=1}^{\mathrm{m}} \mathrm{x}_{i j} \geq \Psi_{\mathrm{j}}^{-1} \delta_{j} \quad(j=1,2, \ldots, n), \\
& \mathrm{x}_{\mathrm{ij}} \geq 0, \forall i, j .
\end{aligned}
$$

Here, we consider the uncertain MOTP under fuzzy multi-choice goal environment i.e., each objective function of the MOTP has some specific fuzzy goals, $g_{t}^{k t}$ for $\mathrm{t}=1,2, \ldots, \mathrm{r}$ for $\mathrm{k}=1,2, \ldots, \mathrm{p}$. According to the problem, the DM can assign the weights to each objective function in such way that it produces a better compromise solution. To do this, we construct a crisp model of the MOTP which is of maximizing type problem whatever the nature of the objective functions of the MOTP.

Again for optimizing the values of the objective functions, the number of fuzzy allocation goals $g_{t}^{t i}$ may not be equal for all the objective functions. If there be only one fuzzy goal $g_{t}^{1}$ for each objective function, then the corresponding mathematical model (i.e., Model 5 ) is derived from Model 4 as follows:

\section{Model 5}

maximize $z=\sum_{t=1}^{r} w_{t} \mu_{t}$,

subject to

$$
\begin{aligned}
& \mathrm{Z}^{\mathrm{t}}=\sum_{\mathrm{i}=1}^{\mathrm{m}} \sum_{\mathrm{j}=1}^{\mathrm{n}}\left[\mathrm{C}_{\mathrm{ij}}^{\mathrm{t}} \pm \mathrm{C}_{\mathrm{ij}}^{\mathrm{t}}\left(1-\mathrm{R}_{\mathrm{ij}}^{\mathrm{t}}\right)\right] \mathrm{x}_{\mathrm{ij},} \\
& \mu_{t} \leq 1-\frac{z^{\mathrm{t}}-\hat{g}_{t}^{1}}{d_{\mathrm{t}}^{1-}} \quad \forall t, \\
& \mu_{t} \leq 1-\frac{g_{\mathrm{r}}^{1}-z^{\mathrm{t}}}{d_{\mathrm{t}}^{1+}} \quad \forall t, \\
& \mu_{t} \geq 0 \quad \forall t, \\
& \sum_{j=1}^{n} \mathrm{x}_{\mathrm{i} j} \leq \theta_{\mathrm{i}}^{-1}\left(1-\gamma_{i}\right)(i=1,2, \ldots, m), \\
& \sum_{i=1}^{m} \mathrm{x}_{i j} \geq \Psi_{j}^{-1} \delta_{j}(j=1,2, \ldots, n), \\
& \mathrm{x}_{\mathrm{ij}} \geq 0 \forall i, j .
\end{aligned}
$$

where $d_{t}^{1-}$ and $d_{t}^{1+}$ are the negative and positive deviations corresponding to the goals $g_{\mathrm{t}}^{1}$ of the objective function $Z^{t}$ respectively.

Now, assuming that if each objective function has two fuzzy aspiration levels, then FMCGP selects any one of these goals in such a way that it provides a better optimal solution. Based on the model of Chang ([3]), the equations; i.e., (6) and (7) reduce to the following form as:

$$
\begin{aligned}
& \mu_{t} \leq 1-\left[\frac{z^{t}-\hat{g}_{t}^{1}}{d_{t}^{1-}} z_{t}^{1}+\frac{z^{k}-\hat{g}_{t}^{2}}{d_{t}^{2-}}\left(1-z_{t}^{1}\right)\right] \quad \forall t, \\
& \mu_{\mathrm{t}} \leq 1-\left[\frac{\hat{g}_{\mathrm{t}}^{1}-z^{\mathrm{t}}}{d_{\mathrm{t}}^{1+}} z_{\mathrm{t}}^{1}+\frac{\hat{g}_{\mathrm{t}}^{2}-z^{\mathrm{t}}}{d_{\mathrm{t}}^{2+}}\left(1-z_{\mathrm{t}}^{1}\right)\right] \quad \forall t \\
& z_{t}^{1}=0 \text { or } 1 \forall i, j \text { and } t \text {. }
\end{aligned}
$$

Here, $d_{t}^{k-}$ and $d_{t}^{h+}$ are the maximum allowable negative and positive deviations respectively for $g_{t}^{k}$ for $k=1,2$.

According to the real-life phenomenon, the objective function may have more than two fuzzy aspiration levels, then we design the corresponding mathematical model in the following way.

So, when each objective function has three fuzzy aspiration levels, FMCGP takes any one of these goals in such a way that it produces to a better optimal solution. Therefore, based on the model of Chang ([3]), the equations (6) and (7) reduce as follows: 
$\mu_{t} \leq 1-\left[\frac{z^{\mathrm{t}}-\hat{g}_{\mathrm{t}}^{1}}{d_{\mathrm{t}}^{1-}} z_{\mathrm{t}}^{1} z_{\mathrm{t}}^{2}+\frac{z^{\mathrm{t}}-\hat{g}_{\mathrm{t}}^{2}}{d_{\mathrm{t}}^{2-}}\left(1-z_{\mathrm{t}}^{1}\right) z_{\mathrm{t}}^{2}+\right.$

$\left.\frac{z^{t}-\hat{g}_{t}^{3}}{d_{t}^{3-}} z_{t}^{1}\left(1-z_{t}^{2}\right)\right] \quad \forall t$

$\mu_{k} \leq 1-\left[\frac{\hat{g}_{\mathrm{t}}^{1}-z^{\mathrm{t}}}{d_{\mathrm{t}}^{1+}} z_{\mathrm{t}}^{1} z_{\mathrm{t}}^{2}+\frac{\hat{g}_{\mathrm{t}-z^{\mathrm{t}}}^{2}}{d_{\mathrm{t}}^{2+}}\left(1-z_{\mathrm{t}}^{1}\right) z_{\mathrm{t}}^{2}+\right.$

$\left.\frac{\hat{g}_{t}^{2}-z^{t}}{d_{t}^{3+}} z_{t}^{1}\left(1-z_{t}^{2}\right)\right] \quad \forall t$

$z_{t}^{1}+z_{t}^{2} \geq 1 \quad \forall t$

$z_{t}^{1}=0$ or $1 \quad \forall t$.

Similarly, $d_{t}^{k-}$ and $d_{t}^{k+}$ are the maximum allowable negative and positive deviations respectively for $g_{t}^{k}$ for $k=1,2,3$.

If $\hat{g}_{t}^{h}(k=1,2, \ldots, p)$ denotes the fuzzy multi-choice goals for the objective functions $Z^{t}(t=1,2, \ldots, r)$, then the linear membership function $\mu_{t}$ for $t^{\text {th }}$ objective function can be defined as follows:

$$
\mu_{t}= \begin{cases}0, & Z^{t} \geq \hat{g}_{t}^{k}+d_{t}^{k+}, \\ 1-\sum_{k=1}^{p} \frac{Z^{t}-\hat{g}_{t}^{k}}{d_{t}^{k-}} F_{k}(B), & \hat{g}_{t}^{k} \leq Z^{t} \leq d_{t}^{k+}+\hat{g}_{t}^{k}, \\ 1, \hat{g}_{t}^{k}=Z^{t}, \\ 1-\sum_{t=1}^{p} \frac{\hat{g}_{t}^{k}-Z^{t}}{d_{t}^{k+}} F_{k}(B), & \hat{g}_{t}^{k}-d_{t}^{k-} \leq Z^{t} \leq g_{t}^{k},\end{cases}
$$

Here, $F_{k}(B)$ indicates a function of binary serial numbers that ensures only one aspiration level must be chosen from each goal (For detailed analysis, one can see ([29]). In general, $d_{t}^{k+}$ and $d_{t}^{k-}$ are the maximum allowable positive and negative deviations respectively from the $\mathrm{k}^{\text {th }}$ aspiration level of the $t^{\text {th }}$ objective function respectively.

It may be noted that, it is not necessary that each objective function has the same number of multi-choice goals. Then Model 5 is developed according to the number of fuzzy goals for the objective functions and then solving, we obtain the aspiration level of each objective function as well as the optimal solution of the MOTP.

\section{Numerical Example}

To test the efficiency of our proposed study, we consider a coal transportation problem, which mainly refers to the MOTP with fuzzy multi-choice goals to the objective functions. The MOTP is designed based on uncertain supply and demand along with the concept of reliability to the cost parameter of the TP.

The DM plans to distribute the coals from three mines M1, M2 and M3 to four Thermal Plants which are situated in the cities, C1, C2, C3 and C4. During the planning, he wishes to optimize the following objective functions as:

- $\quad$ minimize the transportation $\operatorname{cost}\left(\mathrm{Z}^{1}\right)$,

- minimize the toll tax $\left(\mathrm{Z}^{2}\right)$

- maximize the profit $\left(\mathrm{Z}^{\mathrm{a}}\right)$.

According to the market scenario, the DM cannot predict the optimal goals for the objective functions $\mathrm{Z}^{1}, \mathrm{Z}^{2}$ and $\mathrm{Z}^{3}$ in a crisp way. The experience of the DM in the work field, the DM has the ideas about the nature of the objective functions and using this he considers a number of fuzzy goals corresponding to the objective functions. Again, from his experience he guesses the following events.

- The cost $C_{i j}^{1}$ for transporting one unit of goods from the resource $i$ to the destination $j$ and the approximate loss of time of delivery from the sources to the destinations are also known to the DM, for $i=1,2,3$ and $j=1,2,3,4$ respectively.

- The toll tax cost $C_{i j}^{2}$ for transporting one unit of goods from the resource $i$ to the destination $j$ and it is fixed value for $i=1,2,3$ and $j=1,2,3,4$ respectively.

- The profit $C_{i j}^{B}$ for transporting one unit of goods from the resource $i$ to the destination $j$ and the DM made a prediction of approximate loss of time of delivery from the sources to the destinations, for $i=1,2,3$ and $j=1,2,3,4$ respectively.

The data for the transportation costs $C_{i j}^{t}$ for $t=1,2,3$ are represented in Tables 1, 2 and 3.

Table 1. Transportation $\operatorname{cost} C_{i j}^{1}$ (in $\$$ ) and loss of time (in week).

\begin{tabular}{|l|l|l|l|l|}
\hline & C1 & C2 & C3 & C4 \\
\hline M1 & $(20,0.1)$ & $(18,0.1)$ & $(22,0.1)$ & $(24,0.1)$ \\
\hline M2 & $(10,0)$ & $(12,0.2)$ & $(15,0)$ & $(13,0)$ \\
\hline M3 & $(22,0)$ & $(20,0.1)$ & $(24,1)$ & $(23,0.15)$ \\
\hline
\end{tabular}


Table 2. Toll tax $\operatorname{cost} C_{i j}^{2}$ (in $\$$ ) for transportation of goods.

\begin{tabular}{|l|l|l|l|l|}
\hline & C1 & C2 & C3 & C4 \\
\hline M1 & 5 & 6 & 4 & 3 \\
\hline M2 & 6 & 5 & 5 & 4 \\
\hline M3 & 9 & 8 & 8 & 10 \\
\hline
\end{tabular}

Table 3. Cost Parameters $C_{i j}^{a}$ related to profit (in $\$$ ) and loss of time (in week).

\begin{tabular}{|l|l|l|l|l|}
\hline & C1 & C2 & C3 & C4 \\
\hline M1 & $(3,0.1)$ & $(3.5,0.1)$ & $(2.5,0.1)$ & $(5,0.1)$ \\
\hline M2 & $(3,0)$ & $(6,0.2)$ & $(4,0)$ & $(4,0)$ \\
\hline M3 & $(4,0)$ & $(3,0.1)$ & $(4,1)$ & $(5,0.15)$ \\
\hline
\end{tabular}

The supply parameters $a_{1}, a_{2}$ and $a_{3}$ of mines M1, M2 and M3 and the demand parameters $b_{1}, b_{2}, b_{3}$ and $b_{4}$ of cities $\mathrm{C} 1, \mathrm{C} 2, \mathrm{C} 3$ and $\mathrm{C} 4$ follow Normal distribution $N\left(e_{i}^{1}, \sigma_{i}^{1}\right)$, for $i=1,2,3$; and $N\left(e_{j}^{2}, \sigma_{j}^{2}\right)$, for $j=1,2,3,4$ respectively. The data for supply $a_{i}$ and demand $b_{j}$ $\forall i, j$ are presented in Tables 4 and 5 .

Table 4. Supply parameter $a_{i}$ follows Normal distribution $N\left(e_{i}^{1}, \sigma_{i}^{1}\right)$.

\begin{tabular}{|c|c|c|}
\hline M1 & M2 & M3 \\
\hline$(55,4)$ & $(60,5)$ & $(70,4)$ \\
\hline
\end{tabular}

Table 5. Demand parameter $b_{j}$ follows Normal distribution $N\left(\theta_{j}^{2}, \sigma_{j}^{2}\right)$.

\begin{tabular}{|l|l|l|l|}
\hline $\mathrm{C} 1$ & $\mathrm{C} 2$ & $\mathrm{C} 3$ & $\mathrm{C} 4$ \\
\hline$(40,3)$ & $(36,4)$ & $(35,5)$ & $(40,3)$ \\
\hline
\end{tabular}

Again, in the proposed problem, obviously the DM would like to minimize the transportation cost and toll tax cost and maximize the profit. In this situation, the DM expects that the possible expenditure for transportation cost may be any one of fuzzy interval values 2900(100), 4000(100) and 3400(100), here the number within the first bracket denotes the deviations (positive and negative deviations are same here). Again, he expects the expenditure due to toll tax cost either $950(50)$ or $1250(40)$. He also predicts the profit goal may be chosen among the fuzzy values 480(30), 1050(20), 650(50) and 900(50). He wishes to consider the equal weights " 0.3 " to the objective functions $\mathrm{Z}^{1}$ and $\mathrm{Z}^{2}$ and an weight " 0.4 " for the objective function $\mathrm{Z}^{\mathrm{a}}$ respectively. Then the proposed problem takes the following mathematical form as:

\section{Model 6}

$\mathrm{Z}^{1}=\sum_{\mathrm{i}=1}^{\mathrm{a}} \sum_{\mathrm{j}=1}^{4} \mathrm{R}\left[\mathrm{C}_{\mathrm{ij}}^{1}\right] \mathrm{x}_{\mathrm{ij}}, \mathrm{Z}^{1}$ has the goal $[2900(100)$, 4000(100), 3400(100)]

$\mathrm{Z}^{2}=\sum_{\mathrm{i}=1}^{\mathrm{a}} \sum_{\mathrm{j}=1}^{4} \mathrm{C}_{\mathrm{ij}}^{2} \mathrm{x}_{\mathrm{ij}}, \mathrm{Z}^{2}$ has the goal [950(50), 1250(40)]

$\mathrm{Z}^{3}=\sum_{\mathrm{i}=1}^{\mathrm{a}} \sum_{\mathrm{j}=1}^{4} \mathrm{R}\left[\mathrm{C}_{\mathrm{ij}}^{\mathrm{a}}\right] \mathrm{x}_{\mathrm{ij}}, \mathrm{Z}^{\mathrm{a}}$ has the goal $[480(30)$, $1050(20), 650(50), 900(50)]$

subject to

$$
\begin{aligned}
& \mathcal{M}\left(\sum_{j=1}^{n} \mathrm{x}_{i j} \leq a_{i}\right) \geq \gamma_{i}, \quad i=1,2,3, \\
& \mathcal{M}\left(\sum_{i=1}^{m} x_{i j} \geq b_{j}\right) \geq \delta_{j}, \quad j=1,2,3,4, \\
& \mathrm{x}_{\mathrm{ij}} \geq 0 \forall i, j .
\end{aligned}
$$

The uncertain parameters are taken as Normal variable $N(e, \sigma)$ where e is the expectation and $\sigma$ is the standard deviation. The inverse uncertain distribution is defined as $\quad \varphi^{-1}(\mathrm{x})=e+\frac{\sqrt{3} \sigma}{\pi} \ln \left(\frac{1-\mathrm{x}}{\mathrm{x}}\right)$.

Then Model 6 reduces to the following form as:

\section{Model 7}

$\mathrm{Z}^{1}=\sum_{\mathrm{i}=1}^{\mathrm{a}} \sum_{\mathrm{j}=1}^{4}\left(\mathrm{C}_{\mathrm{ij}}^{1}+\mathrm{C}_{\mathrm{ij}}^{1}\left(1-\mathrm{R}_{\mathrm{ij}}^{1}\right)\right) \mathrm{x}_{\mathrm{ij}}$,

$\mathrm{Z}^{1}$ has the goal [2900(100), 4000(100), 3400(100)],

$\mathrm{Z}^{2}=\sum_{\mathrm{i}=1}^{\mathrm{a}} \sum_{\mathrm{j}=1}^{4} \mathrm{C}_{\mathrm{ij}}^{2} \mathrm{x}_{\mathrm{ij}}$,

$\mathrm{Z}^{2}$ has the goal $[950(50), 1250(40)]$,

$\mathrm{Z}^{3}=\Sigma_{i=1}^{3} \Sigma_{j=1}^{4}\left(\mathrm{C}_{\mathrm{ij}}^{\mathrm{B}}-\mathrm{C}_{\mathrm{ij}}^{\mathrm{B}}\left(1-\mathrm{R}_{\mathrm{ij}}^{\mathrm{B}}\right)\right) \mathrm{x}_{\mathrm{ij}}$,

$\mathrm{Z}^{\mathrm{a}}$ has the goal $[480(30), 1050(20), 650(50), 900(50)]$,

subject to

$\sum_{j=1}^{4} x_{i j} \leq e_{i}^{1}+\frac{\sqrt{3} \sigma_{i}^{1}}{\pi} \ln \left(\frac{1-\gamma_{i}}{\gamma_{i}}\right) \quad(i=1,2,3)$,

$\sum_{\mathrm{i}=1}^{3} \mathrm{x}_{\mathrm{ij}} \geq e_{j}^{2}+\frac{\sqrt{ } \sigma_{j}^{2}}{\pi} \ln \left(\frac{s_{j}}{1-\delta_{j}}\right) \quad(\mathrm{j}=1,2,3,4)$,

$\mathrm{x}_{\mathrm{ij}} \geq 0 \forall i, j$.

Here, $R_{i j}^{1}$ and $R_{i j}^{3}$ are the reliability of completing the job of transportation in time, They are taken as function of the decision variables and the expected loss time for completion the work. The source capacity $\left(a_{i}\right)$ is uncertain in nature, but we use this value for maximum priority in $R_{i j}^{1}$ and $R_{i j}^{B}$. Let us assume the confidence levels be $\gamma_{i}=0.85$ and $\delta_{j}=0.9, \quad \forall i=1,2,3$ and $j=1,2,3,4$. 
Finally, the proposed MOTP is converted to a single objective problem using the goals as prescribed by the DM and we obtain the following model as:

\section{Model 8}

$$
\begin{aligned}
& \text { maximize } \quad z=0.3 \mu_{1}+0.3 \mu_{2}+0.4 \mu_{3} \\
& \text { subject to } \\
& \mathrm{Z}^{1}=\left(40-20 e^{-\frac{0.1}{55} \mathrm{x}_{11}}\right) \mathrm{x}_{11}+\left(36-18 e^{-\frac{0.1}{55} \mathrm{x}_{12}}\right) \mathrm{x}_{12} \\
& \quad+\left(44-22 e^{-\frac{0.1}{55} \mathrm{x}_{13}}\right) \mathrm{x}_{13}+\left(26-13 e^{-\frac{0.1}{55} \mathrm{x}_{14}}\right) \mathrm{x}_{14} \\
& \quad+\left(20-10 e^{-\frac{a}{60} \mathrm{x}_{21}}\right) \mathrm{x}_{21}+\left(24-12 e^{-\frac{0.2}{60} \mathrm{x}_{22}}\right) \mathrm{x}_{22} \\
& \quad+\left(30-15 e^{-\frac{0}{60} \mathrm{x}_{23}}\right) \mathrm{x}_{23}+\left(26-13 e^{-\frac{0}{60} \mathrm{x}_{24}}\right) \mathrm{x}_{24} \\
& +\left(44-22 e^{-\frac{0}{70} \mathrm{x}_{31}}\right) \mathrm{x}_{31}+\left(40-20 e^{-\frac{0.1}{70} \mathrm{x}_{32}}\right) \mathrm{x}_{32} \\
& +\left(48-24 e^{-\frac{1}{70} x_{33}}\right) \mathrm{x}_{33}+\left(46-23 e^{-\frac{0.15}{70} x_{34}}\right) \mathrm{x}_{34}
\end{aligned}
$$$$
\mathrm{z}^{2}=5 \mathrm{x}_{11}+6 \mathrm{x}_{12}+4 \mathrm{x}_{13}+3 \mathrm{x}_{14}+6 \mathrm{x}_{21}+5 \mathrm{x}_{22}+
$$$$
5 \mathrm{x}_{23}+4 \mathrm{x}_{24}+9 \mathrm{x}_{31}+8 \mathrm{x}_{32}+8 \mathrm{x}_{33}+10 \mathrm{x}_{34} \text {, }
$$$$
\mathrm{Z}^{3}=3 e^{-\frac{0.1}{55} \mathrm{x}_{11}} \mathrm{x}_{11}+3.5 e^{-\frac{0.1}{55} \mathrm{x}_{12}} \mathrm{x}_{12}+2.5 e^{-\frac{0.1}{55} \mathrm{x}_{13}} \mathrm{x}_{13}+
$$$$
5 e^{-\frac{0.1}{55} x_{14}} \mathrm{x}_{14}+3 e^{-\frac{0}{60} x_{21}} \mathrm{x}_{21}+6 e^{-\frac{0.2}{60} x_{22}} \mathrm{x}_{22}+
$$$$
4 e^{-\frac{0}{60} x_{23}} x_{23}+4 e^{-\frac{0}{60} x_{24}} x_{24}+4 e^{-\frac{0}{70} x_{31}} x_{31}+
$$$$
3 e^{-\frac{0.1}{70} x_{32}} x_{32}+4 e^{-\frac{1}{70} x_{33}} x_{33}+5 e^{-\frac{0.15}{70} x_{34}} x_{34} \text {, }
$$$$
\mu_{1} \leq 1-\left[\frac{z^{1}-2900}{100} \quad z_{1}^{1} z_{1}^{2}+\frac{z^{1}-4000}{100} \quad\left(1-z_{1}^{1}\right) z_{1}^{2}\right.
$$$$
\left.+\frac{z^{1}-9400}{100} z_{1}^{1}\left(1-z_{1}^{2}\right)\right] \text {, }
$$$$
\mu_{1} \leq 1-\left[\frac{2900-z^{1}}{100} z_{1}^{1} z_{1}^{2}+\frac{4000-z^{1}}{100}\left(1-z_{1}^{1}\right) z_{1}^{2}\right.
$$$$
\left.+\frac{3400-z^{1}}{100} z_{1}^{1}\left(1-z_{1}^{2}\right)\right]
$$$$
\mu_{2} \leq 1-\left[\frac{z^{2}-950}{50} z_{2}^{1}+\frac{z^{2}-1250}{40}\left(1-z_{2}^{1}\right)\right],
$$$$
\mu_{2} \leq 1-\left[\frac{950-z^{2}}{50} z_{2}^{1}+\frac{1250-z^{2}}{40}\left(1-z_{2}^{1}\right)\right],
$$$$
\mu_{3} \leq 1-\left[\frac{z^{3}-490}{30} z_{3}^{1} z_{3}^{2}+\frac{z^{3}-1050}{20}\left(1-z_{3}^{1}\right) z_{3}^{2}+\frac{z^{3}-650}{50}\right.
$$$$
\left.z_{3}^{1}\left(1-z_{3}^{2}\right)+\frac{z^{3}-900}{50}\left(1-z_{3}^{1}\right)\left(1-z_{3}^{2}\right)\right] \text {, }
$$$$
\mu_{3} \leq 1-\left[\frac{490-z^{3}}{30} z_{3}^{1} z_{3}^{2}+\frac{1050-z^{3}}{20}\left(1-z_{3}^{1}\right) z_{3}^{2}+\frac{650-z^{3}}{50}\right.
$$$$
\left.z_{3}^{1}\left(1-z_{3}^{2}\right)+\frac{900-z^{3}}{50}\left(1-z_{3}^{1}\right)\left(1-z_{3}^{2}\right)\right],
$$

$$
z_{1}^{1}+z_{1}^{2} \geq 1
$$$$
z_{k}^{t}=0 \text { or } 1 \quad \forall k, t
$$

$$
\begin{aligned}
& 0 \leq \mu_{k} \leq 1 \quad k=1,2,3, \\
& \mathrm{x}_{11}+\mathrm{x}_{12}+\mathrm{x}_{13}+\mathrm{x}_{14} \leq 51.175, \\
& \mathrm{x}_{21}+\mathrm{x}_{22}+\mathrm{x}_{23}+\mathrm{x}_{24} \leq 55.218, \\
& \mathrm{x}_{31}+\mathrm{x}_{32}+\mathrm{x}_{33}+\mathrm{x}_{34} \leq 66.175, \\
& \mathrm{x}_{11}+\mathrm{x}_{21}+\mathrm{x}_{31} \geq 43.634, \\
& \mathrm{x}_{12}+\mathrm{x}_{22}+\mathrm{x}_{32} \geq 40.486, \\
& \mathrm{x}_{13}+\mathrm{x}_{23}+\mathrm{x}_{33} \geq 41.057, \\
& \mathrm{x}_{14}+\mathrm{x}_{24}+\mathrm{x}_{34} \geq 43.634, \\
& \mathrm{x}_{\mathrm{ij}} \geq 0 \forall i, j .
\end{aligned}
$$

Solving Model 8 by using LINGO software, we derive the following solution: Optimum value of $z=0.82$.

Hence, the optimal values of the objective functions are: $Z^{1}=3400.00, Z^{2}=980.13$ and $Z^{3}=650$.

The allocations are made as follows:

$\mathrm{x}_{11}=3.12 ; \mathrm{x}_{12}=0.0 ; \mathrm{x}_{13}=18.95 ; \mathrm{x}_{14}=29.10$;

$\mathrm{x}_{21}=11.26 ; \mathrm{x}_{22}=25.07 ; \mathrm{x}_{23}=4.36 ; \mathrm{x}_{24}=14.54$;

$\mathrm{x}_{31}=29.26 ; \mathrm{x}_{\mathrm{az}}=15.42 ; \mathrm{x}_{\mathrm{a3}}=17.74 ; \mathrm{x}_{\mathrm{a} 4}=0.0$;

Selected goal for the objective functions are as follows: $3400(100)$ for $Z^{1}, 950(50)$ for $Z^{2}$ and 650(50) for $Z^{3}$.

\section{Sensitivity Analysis}

Here, we present a study on the MOTP with fuzzy multi-choice goals for each objective functions under uncertain environment. Especially, here, we incorporate the situation of cost reliability with the cost parameters due to delay of delivery of goods before/after schedule time. The numerical example is presented the applicability of proposed methodology for solving the MOTP with fuzzy multi-choice goals and uncertain supply and demand parameters. Usually, the MOTP having the objective functions are of either maximization type or minimization type, but here, the aim of the DM is not likely to the solution of traditional MOTP. This work presents the uncertainty under the expectation of an upcoming event and also proposes to fix-up the goals for each of the objective functions for the DM corresponding to the possible optimal values. On the other hand, time is very much important for transporting the goods to real-life transportation problems, so the decision making under time consideration and cost reliability provide a proper way of selecting the goals for the objective functions.

In the proposed problem, if the DM wishes to find the optimal solution of the objective function under traditional way like goal programming approach, then certainly he may consider minimum goals for first two objective functions $Z^{1}$ and $Z^{2}$ i.e., 2900 and 950 respectively and for the profit objective function $Z^{3}$, the maximum goal is 1050. Considering these goals 
corresponding to the objective functions, we attempt to solve the proposed problem using goal programming, but we cannot obtain any feasible solution through GP. Again we have tested that, if the DM considers the goal values 4000 for the objective function $\mathrm{Z}^{1}$ and 1250 for the objective function $Z^{2}$ then the DM calculated the maximum profit goal 1050 for the objective function $\mathrm{Z}^{3}$ through goal programming approach. Basically, in most cases, the customers pay the transportation cost and toll tax cost, if the costs increase then the DM may loose the customer in near future which happens due to maximum profit goal of the DM. As a result the optimal solution through GP has not produced a better result in both cases. To test the utility of our proposed method, we consider multi-choice goals $(2900,4000,3400)$ for $Z^{1}$; $(950,1250)$ for $Z^{2} ;(480,1050,650,900)$ for $Z^{3}$ and solving the problem by revised multi-choice goal programming (RMCGP) approach, we see that the selected optimal goal values are 2900 for $Z^{1}$; 950 for $\mathrm{Z}^{2} ; 480$ for $\mathrm{Z}^{3}$. In this case the DM achieves the solution in which selected profit goal is minimum value and it is not satisfactory to the DM. So, the proposed approach is a better than GP or RMCGP technique for selecting optimal goals. Hence, in that situation, the DM cannot fix the goals of the objective functions using the existing techniques, whereas our presented methodology can give a better solution as well as a better selection of goals for the MOTP through uncertain environment.

\section{Conclusion}

In this paper, we have analyzed the real-life MOTP through the concept of reliability and uncertain environments. We have proposed a new kind of uncertainty on cost parameter based on the concept reliability. Beside this, we have established the MOTP under the consideration of fuzzy multi-choice goals to the objective functions and the supply and demand are taken as uncertain in nature. A solution procedure for solving the MOTP; and the selection of goals for the objective functions have been discussed by taking a real-life example. The obtained results have indicated that the proposed approach has a better impact to solve the MOTP under uncertain environment; and it has the advantages of selection of goals for real-life decision making problems under uncertain environment. This method is not only proposed the subjective preference into real-life decision-making problems, but also can realize the better selection of goals to the objective functions.

The proposed method has the following limitations for formulating the mathematical model. Firstly, the value of delay time or over time of transportation in the reliability function should have to be taken in a unit scale and in this case the time $\tau \leq 1$, otherwise, the value of reliability function may not provide for selecting the goals and optimal solution. Another important factor is that at least one multi-choice fuzzy goals for each of the objective functions $\left(z^{\mathrm{i}}\right)$ must intersect the interval range $\left[\min Z^{\mathrm{i}}, \max Z^{\mathrm{i}}\right] \forall i$ for getting the optimal solution of the MOTP, otherwise it produces infeasible solution. Thus for a better result of the MOTP, the above two restrictions must be taken into care by the DM.

There are many directions for future study. One main direction is to design and extend the proposed study to a class of MOTP where uncertain coefficients are presented in both objective functions and constraints. Another direction is that this study can be implemented to find the solution of managerial problems occurred in the field of inventory, supply chain management, etc. when they are connected with transportation problem.

\section{Acknowledgment}

The first author is highly acknowledged to the University Grants Commission of India for supporting the financial assistance to continue this research work under JRF(UGC) scheme: Sanction letter number [F.17130/1998(SA-I)] dated 26/06/2014. Also, last author wants to thank the financial help received from the Spanish Govern under the project TIN2014-55024-P as well as from the Andalusian Govern under project TIC8001 (both financed with FEDER funds).

The authors are very much thankful to Area Editor Prof. R. Dou and the anonymous reviewers for their constructive comments to improve the quality of the paper.

\section{References}

1. F. L. Hitchcock, The distribution of a product from several sources to numerous localities, Journal of Math. Phys, 20 (1941), 224-230.

2. T.C. Koopmans, Optimum utilization of the transportation System, Econometrica, 17 (1949) 136-146.

3. A. Charnes and W. W. Cooper, Management models and industrial applications of linear programming, Management Science, 4(1) (1957), 38-91.

4. R. S. Garfinkel and M.R. Rao, The bottleneck transportation problem, Naval Research Logistics Quarterly, 18 (1971), 465-472. 
5. J.M. Cadenas, and J.L. Verdegay, Using ranking functions in multi-objective fuzzy linear programming, Fuzzy sets and systems, 111 (1) (2000), 47-53.

6. F Abd El-Wahed Waiel, A multi-objective transportation problem under fuzziness, Fuzzy Sets and Systems, 117 (2001), 27-33.

7. D.R. Mahapatra, S.K. Roy, M.P. Biswal. Stochastic based on multi-objective transportation problems involving normal randomness, Advanced Modeling and Optimization, 12 (2) (2010), 205-223.

8. D.R Mahapatra, S.K. Roy and M.P. Biswal. Multiobjective stochastic transportation problem involving lognormal, Journal of Physical Sciences, 14 (2010), 63-76.

9. A. Gupta and A. Kumar, A new method for solving linear multi-objective transportation problems with fuzzy parameters. Applied Mathematical Modelling, 36 (2012), 1421-1430.

10. A. Ebrahimnejad, A simplified new approach for solving fuzzy transportation problems with generalized trapezoidal fuzzy numbers, Applied Soft Computing, 19 (2014), 171-176.

11. F. Jiménez and J.L. Verdegay, Solving fuzzy solid transportation problems by an evolutionary algorithm based parametric approach, European Journal of Operational Research, 117 (3) (1999), 485-510.

12. M. Delgado, J.L. Verdegay and M.A. Vila, A general model for fuzzy linear programming, Fuzzy Sets and systems, 29 (1) (1989), 21-29.

13. D.R. Mahapatra, S.K. Roy and M.P. Biswal, Multichoice stochastic transportation problem involving extreme value distribution, Applied Mathematical Modelling, 37(4) (2013), 2230-2240.

14. G. Maity and S.K. Roy, Solving a multi-objective transportation problem with nonlinear cost and multi-choice demand, International Journal of Management Science and Engineering Management, 11(1) (2016), 62- 70.

15. S. Midya and S.K. Roy, Single-sink, fixed-charge, multi-objective, multi-index stochastic transportation problem, American Journal of Mathematical and Management Sciences, 33 (2014), 300-314.

16. S.K. Roy, D.R. Mahaparta and M.P. Biswal, Multichoice stochastic transportation problem with exponential distribution, Journal of Uncertain Systems, 6 (3) (2012), 200-213.

17. B. Liu, Uncertainty Theory, 2nd ed., SpringerVerlag, Berlin, 2007.

18. B. Liu, Fuzzy Process, Hybrid Process and Uncertain Process, Journal of Uncertain Systems, 2 (2008), 3-16.

19. B. Liu, Uncertaint Theory: A Branch of Mathematics for Modeling Human Uncertainty, Springer-Verlag, Berlin, 2010.
20. X. Gao, Some Properties of Continuous Uncertain Measure, International Journal of Uncertainty, Fuzziness and Knowledge-Based Systems, 17 (2009), 419-426.

21. B. Liu, Theory and Practice of Uncertain Programming, 2nd ed., Springer-Verlag, Berlin, 2009.

22. A. Charnes, W.W. Cooper and R.O. Ferguson, Optimal estimation of executive compensation by linear programming, Management Science, 1 (1955), 138-151.

23. C.T. Chang, Multi-choice goal programming, Omega, 35 (2007), 389-396.

24. C.N. Liao, Formulating the multi-segment goal programming, Computers \& Industrial Engineering, 56(1) (2009), 138-141.

25. S.M. Lee, Goal programming for decision analysis, Auerbach Publishers: Philadelphia, 1972.

26. G. Maity and S.K. Roy, Solving multi-choice multiobjective transportation problem: utility function approach, Journal of Uncertainty Analysis and Applications, Doi:10.1186/2195-5468-2-11, 2014.

27. R. Narasimhan, Goal programming in a fuzzy environment, Decision Sciences, 11 (1980), 325336.

28. M. Tamiz, D.F. Jones and C. Romero, Goal programming for decision making: an overview of the current state-of-the-art, European Journal of Operational Research, 111(3) (1998), 569-581.

29. C.T. Chang, Revised multi-choice goal programming, Applied Mathematical Modelling, 32 (2008), 2587-2595.

30. B. B. Tabrizi, K. Shahanaghi and S. Jabalameli, Fuzzy multi-choice goal programming, Applied Mathematical Modelling, 36 (2012), 1415-1420.

31. S.K. Roy, Multi-choice stochastic transportation problem involving Weibull distribution, International Journal of Operational Research, 21(1) (2014), 38-58.

32. G. Maity, S.K. Roy, Solving multi-objective transportation problem with interval goal using utility function approach, International Journal of Operational Research, 27(4) (2016), 513-529.

33. S.K. Roy and D.R. Mahapatra, Multi-objective interval-valued transportation probabilistic problem involving log-normal, International Journal of Mathematics and Scientific Computing, 1(2) (2011), 14-21.

34. E. B. Richard, P. Frank and C.H. Larry, Mathematical theory of reliability. John Wiley and Sons, Inc. New York. London. Sydney, Third edition, 1967. 